\title{
Collision Induced Decomposition of Peptides. Choice of Collision Parameters*
}

\author{
Ivan Haller, Urooj A. Mirza, and Brian T. Chait \\ Mass Spectrometry Laboratory, The Rockefeller University, 1230 York Avenue, New York, New York, USA
}

Collision-induced dissociation product ion spectra of a series of doubly charged tryptic peptide ions produced by electrospray ionization were obtained by triple-quadrupole tandem mass spectrometry. The sequence information content of the product ion spectra was explored as a function of collision energy and collision-cell gas pressure for parent ions with molecular masses ranging from 300 to $2000 \mathrm{u}$. The energy range (at a given pressure) in which the degree of fragmentation is acceptable was found to be narrow for parent ions of a given mass, and the optimal collision energy was observed to exhibit a strong linear correlation with parent ion mass. This observed correlation opens the way for on-line software-controled selection of optimal mass spectrometric conditions in the enzymatic digestion-liquid chromatography-tandem mass spectrometric strategy of amino acid sequencing of proteins. (J Am Soc Mass Spectrom 1996, 7, 677-681)

A potentially powerful strategy for obtaining amino acid sequence information from proteins utilizes the combination of tryptic digestion followed by liquid chromatography-electrospray ionization tandem mass spectrometry of the resulting peptides $[1,2]$. Under conditions customarily used with triple quadrupole instruments (argon collision gas, $<100-\mathrm{V}$ ion acceleration potential), scission of the peptide bond tends to dominate the observed fragmentation pathways [3-5]. Thus, for example, for doubly charged tryptic peptides, the fragmentation spectrum consists mostly of two easily identifiable series of ions - the $b$ and the $y$ series [6]. These fragment ions can be used to infer the amino acid sequence of the parent ion. Fragmentation at other positions of the peptide chain, multiple cleavages, and losses of water and ammonia complicate the interpretation of the spectra for sequence determination. It is therefore of interest to ascertain the conditions under which one can expect to obtain collision-induced dissociation (CID) spectra with the highest information content. Although numerous investigations have addressed the dependence of the fragmentation behavior on the nature of the peptide [7-10] (i.e., the composition and sequence of the amino acid residues) and the identity of the collision partner $[11,12]$, there is relatively little information available on how the quality and the information content of the spectra depend on the collision energy [12-16].

In this article, we first present measurements of the efficiency of collision-induced decomposition (at a fixed

Address reprint requests to Dr. Brian T. Chait, The Rockefeller University, 1230 York Avenue, New York, NY 10021.

* Presented in part at the 42nd ASMS Conference on Mass Spectrometry and Allied Topics, Chicago, IL May 29-June 3, 1994. collision gas pressure) of a doubly protonated octapeptide as a function of collision energy. The results are used to propose a technique for selection of the optimal collision energy, that is, the collision energy that maximizes the information content of the fragmentation spectrum. We then present measurements of the energy dependence of fragmentation, at three gas pressures, of 18 doubly protonated tryptic peptides. Finally, we discuss an observed correlation between optimal collision energy and peptide mass. Our findings suggest a straightforward method of on-line softwarecontroled selection of optimal tandem mass spectrometry conditions for direct liquid chromatographytandem mass spectrometry (LC-MS/MS) sequencing of proteins.

\section{Experimental}

Peptides (see Table 1) were purchased from Sigma Chemical Co. (St. Louis, MO) and were used without further purification. The peptides were introduced as $10-\mu \mathrm{M}$ solutions in 1:1:0.1 (v/v/v) water/methanol/acetic acid mixture via a syringe pump, at a flow rate of $2 \mu \mathrm{L} / \mathrm{min}$, through the electrospray interface of a Finnigan TSQ 700 triple quadrupole mass spectrometer (Finnigan-MAT, San Jose, CA). The voltages on the hardware elements along the ion-path in the TSQ 700 are, in general, dynamically adjusted under computer control to maximize ion transmission at the mass-tocharge ratio selected. To ensure that the internal energy of the ions remains reproducibly small, voltages on the ion focusing elements in the pre-skimmer and the octapole ion transfer regions were carefully controled over the entire set of measurements. Thus, in the pre-skimmer region ( $p=0.5-1.0$ torr), the poten- 
Table 1. The amino acid sequence, mass, and the obtained fitting parameters for the peptides used

\begin{tabular}{|c|c|c|c|c|c|c|c|}
\hline \multirow[b]{2}{*}{ Amino acid sequence } & \multirow{2}{*}{$\begin{array}{l}\text { Molecular } \\
\text { mass }\end{array}$} & \multicolumn{3}{|c|}{$V_{\text {opt }}(V)$ at (mtorr) } & \multicolumn{3}{|c|}{ Slope $b\left(V^{-1}\right)$ at (mtorr) } \\
\hline & & 2.7 & 1.5 & 0.88 & 2.7 & 1.5 & 0.88 \\
\hline$\overline{T S K}$ & 334.4 & 6.8 & 7.23 & 11.3 & 0.83 & 0.71 & 0.30 \\
\hline PFGK & 447.5 & 9.82 & 10.3 & 13.9 & 0.74 & 0.69 & 0.48 \\
\hline VESSK & 548.6 & 10.0 & 10.7 & 15.2 & 0.76 & 0.71 & 0.31 \\
\hline SIGSL AK & 674.8 & 8.9 & 9.4 & 13.6 & 0.64 & 0.66 & 0.52 \\
\hline VHLTP VEK & 922.1 & 17.8 & 19.4 & 30.6 & 0.50 & 0.50 & 0.13 \\
\hline KROHP GKR & 1006.2 & 27.3 & 30.5 & 45.7 & 0.40 & 0.49 & 0.23 \\
\hline PHPFH FFVYK & 1318.5 & 21.1 & 23.6 & 33.9 & 0.47 & 0.42 & 0.34 \\
\hline QATVG DVNTD RPGLL DLK & 1912.1 & 38.3 & 44.6 & 71.2 & 0.47 & 0.43 & 0.12 \\
\hline GGR & 288.3 & 5.17 & 5.98 & 8.0 & 0.55 & 0.75 & 0.42 \\
\hline GGYR & 451.5 & 6.90 & 7.91 & 11.1 & 0.44 & 0.51 & 0.34 \\
\hline YIGSR & 594.7 & 8.27 & 8.98 & 13.2 & 0.73 & 0.65 & 0.41 \\
\hline HLGLA R & 665.8 & 13.7 & 14.7 & 19.8 & 0.59 & 0.69 & 0.42 \\
\hline ASHLG LAR & 824.0 & 15.4 & 16.6 & 23.7 & 0.73 & 0.65 & 0.41 \\
\hline PPGFS PFR & 904.1 & 18.4 & 19.4 & 27.2 & 0.59 & 0.59 & 0.25 \\
\hline RPPGH SPFR & 1060.3 & 26.2 & 29.4 & 46.1 & 0.40 & 0.38 & 0.14 \\
\hline HSDAV FTDNY TR & 1425.5 & 27.6 & 31.5 & 47.5 & 0.56 & 0.51 & 0.13 \\
\hline EGVND NEEGH FSAR & 1570.6 & 27.9 & 30.4 & 53.5 & 0.30 & 0.39 & 0.11 \\
\hline ADSGE GDFLA EGGGV R & 1536.6 & 23.8 & 27.7 & 41.9 & 0.50 & 0.51 & 0.24 \\
\hline
\end{tabular}

tials (relative to the grounded skimmer) on the heated capillary $\left(T=200{ }^{\circ} \mathrm{C}\right)$ and on the tube lens were maintained constant at 20 and $74 \mathrm{~V}$, respectively, for $20<m / z<300$. Above $m / z 300$ these potentials were increased linearly by 2.5 and $5.7 \mathrm{~V}$, respectively, for each 100 increase in mass-to-charge ratio. Changes of $10 \mathrm{~V}$ in the capillary voltage and $30 \mathrm{~V}$ in the tube lens voltage, although they greatly reduced ion transmission, caused no apparent change in the ion excitation as measured by the ratios of product ions to parent ion. In the differentially pumped mass spectrometry entrance region ( $p \cong 1$ mtorr) the offset voltage of the guiding octapole was maintained constant at $-0.2 \mathrm{~V}$ for all masses. The resolution of both quadrupoles were maintained at $2.5 \mathrm{u}$ full width at half maximum. The present experiments were performed by using spectral accumulation times of $48 \mathrm{~s}$. Argon (Matheson, East Rutherford, NJ; prepurified grade) was used as the collision gas. The collision gas pressure in the collision cell was set by a needle valve in the flow line and was measured by a Granville-Phillips (Boulder, $\mathrm{CO})$ convection gauge whose zero was frequently checked. Readings are reproducible (but not necessarily accurate) to 0.01 mtorr.

\section{Results and Discussion}

\section{Fragmentation Efficiency of an Octapeptide}

Figure 1 shows the CID mass spectra taken at three different collision energies of the doubly protonated ion of the octapeptide ASHLGLAR at a fixed collision gas pressure of 1.5 mtorr. The spectrum in Figure 1a was obtained with the collision-cell offset voltage $V_{c}$

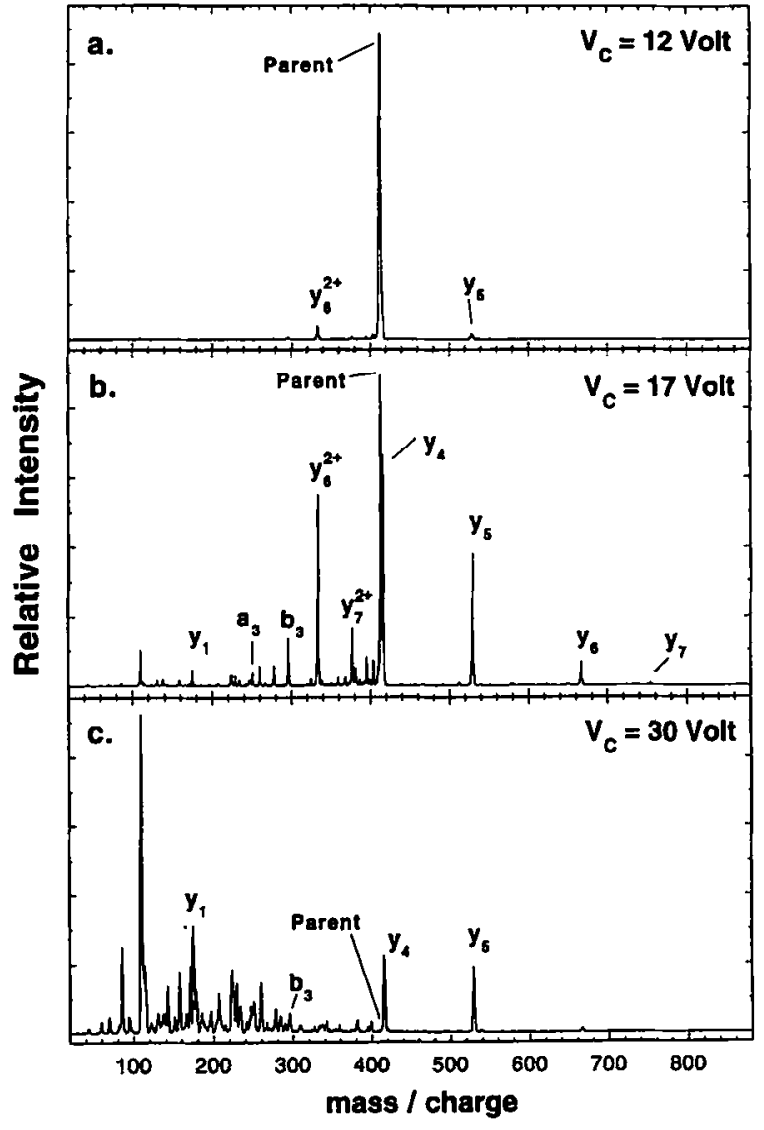

Figure 1. CID spectra of the octapeptide ASHLGLAR at three different acceleration voltages. Note the increase in the relative abundances of smaller fragments in the highest energy spectrum. 
held at $12.0 \mathrm{~V}$. The kinetic energy of the ions in the laboratory frame is $E_{\text {lab }}=z e V_{c}$, where $z$ is the number of charges on the ion; in this case $E_{\text {lab }}=24 \mathrm{eV}$. At this collision energy only a few product ion peaks are observed. The optimum sequence information was at $V_{\mathrm{c}}=17.0 \mathrm{~V}\left(E_{\mathrm{lab}}=34 \mathrm{eV}\right)$, where easily identifiable peaks yielded unambiguous sequence information for five out of the eight amino acid residues (Figure 1b). Higher values of $V_{c}$ (Figure 1c) result in excessive fragmentation, indicated by the diminution of the higher molecular mass fragments and the simultaneous elevation of low mass ions. These low mass fragment ions are more difficult to interpret because they involve secondary fragmentation of the $y$ and $b$ series product ions.

In Figure 2 the measured abundances of the doubly protonated octapeptide ion and four representative major fragment ions are shown as a function of the collision-cell offset voltage $V_{c}$. We make three observations from these measurements: (1) The parent ion intensity decreases with increasing $V_{c}$. (2) Abundances of fragment ions that are useful for sequencing (Figure $2 \mathrm{a}, \mathrm{b}$ and $\mathrm{c}$ ) all go through a maximum and, within the experimental error, the maxima occur at the same voltage. (3) Ions that result from multiple fragmentation, such as the histidine immonium ion shown in

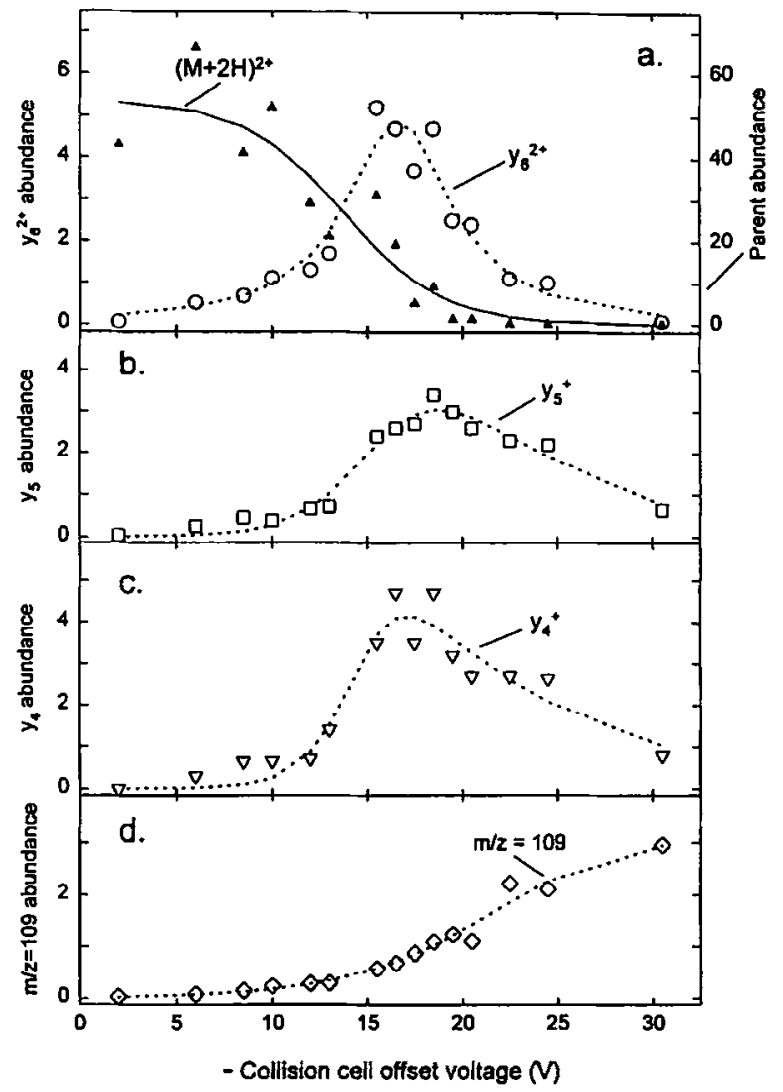

Figure 2. Abundances of the parent ion (filled triangles, scale on right) and the highest intensity product ions in the CID of ASHLGLAR as a function of ion acceleration voltage. The curves are to guide the eye.
Figure $2 \mathrm{~d}$, continue to increase in abundance and saturate at a few volts above the maxima of the primary fragment ions.

The most informative fragmentation spectrum is obtained when the primary fragment ions approach their maximum abundances. To be able to compare optimal conditions for fragmentation of a large number of different peptides, we searched for an objective criterion that quantitates in a simple manner the offset voltage that gives the optimum sequence information. We observe from Figure 2 that the maxima in the product ion peaks occur at a voltage where the sum of the intensities of the three strongest fragment ions reaches about 1.5 times the remaining parent ion intensity. For the purposes of the present study, we define the conversion ratio $R$ as the sum of the abundances of the three strongest fragment ions in the CID spectrum divided by the remaining parent ion abundance. The optimal collision energy $V_{\text {opt }}$ is defined as the collision-cell offset voltage at which the conversion ratio equals 1.5 .

In Figure 3, the conversion ratio is plotted on a semilogarithmic scale versus the offset voltage. By plotting the conversion ratio rather than the absolute fragment ion intensities, we diminish effects that arise from fluctuations in the electrospray ionization yield. The observed exponential dependence indicates that the decomposition reaction is driven by a buildup (via multiple collisions) of internal energy that is approximately proportional to the ion kinetic energy. Other factors are likely to enter into the overall fragmentation efficiency (e.g., the collision cross section and the time available for decomposition); however, the present experimental results do not allow us to evaluate the relative contributions of these other factors.

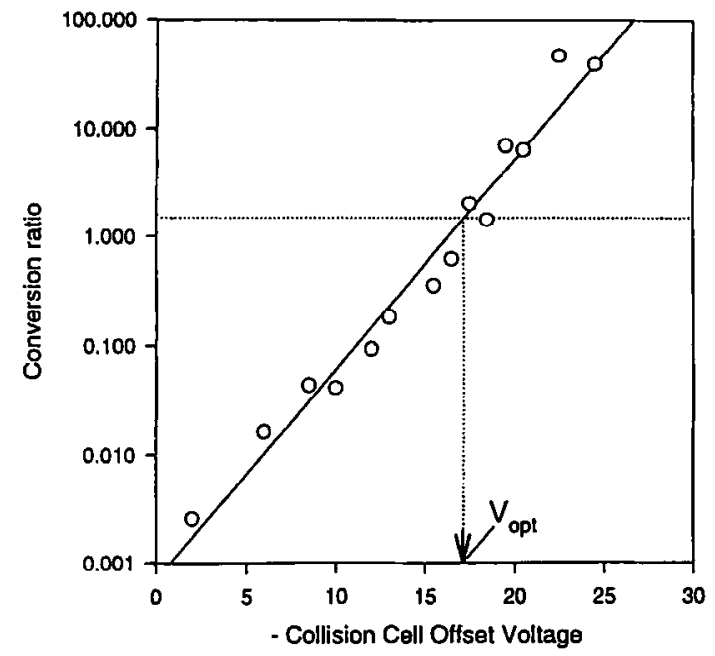

Figure 3. The sum of the abundances of the three strongest fragment ions in the CID spectrum of ASHLGLAR divided by the remaining parent ion abundance (conversion ratio) versus the acceleration voltage. The definition of $V_{\text {opt }}$ is shown by the dotted lines. 


\section{Dependence of the Optimal Collision Energy on Peptide Mass}

We have measured the collision-induced dissociation spectra of doubly protonated* ions of the 18 peptides listed in Table 1 (varying in size from 3 to 18 residues) at collision-cell offset voltages close to $V_{\text {opt }}$. The peptides were of the type that are normally expected from complete or partial trypsin digestion of proteins, that is, their carboxyl termini were lysine (first eight entries in Table 1) or arginine (remaining entries). For each peptide, spectra were recorded at three different collision-cell offset voltages (that bounded $V_{\text {opt }}$ ) at each of three different pressures $(0.88,1.5$, and 2.7 mtorr), which required nine separate measurements on each peptide.

The energy dependence of the fragmentation for all the peptides studied (at each pressure) was qualitatively similar to that of the octapeptide ASHLGLAR discussed previously; that is, the conversion ratio varied exponentially with collision-cell offset voltage over the narrow range of energies investigated, and the $R=1.5$ criterion chosen agreed with more subjective (but nevertheless important) criteria, such as maintaining a good balance between peaks that arise from single versus multiple cleavage reactions of peptide ions. Figure 4 shows a representative set of conversion ratio versus $V_{c}$ data for one of the 18 peptidesHLGLAR. The figure also illustrates our general observation that the conversion ratio is a strong function of the collision gas pressure. To derive the optimum collision-cell offset voltage $V_{\text {opt }}$, for each peptide, the data at each pressure were fitted to the equation

$$
R=1.5 \times \exp \left[b\left(V_{\mathrm{c}}-V_{\text {opt }}\right)\right]
$$

where $R$ is the conversion ratio and $V_{\mathrm{c}}$ is the collision-cell offset voltage. This equation is in accordance with our definition of the optimal acceleration voltage and it reflects the exponential dependence observed in Figures 3 and 4 and in the data for the remaining 16 peptides (not shown). The fitting parameters $V_{\text {opt }}$ and $b$, obtained for the 18 peptides investigated, are given in Table 1.

When we examine $V_{\text {opt }}$ obtained from the 18 peptides studied, we observe a strong linear correlation of $V_{\text {opt }}$ with the size of the parent peptide. Figure 5 shows the dependence of the optimal offset voltage on parent ion molecular mass. $V_{\text {opt }}$ is observed to increase linearly with molecular mass for each of the three pressures used in the measurements and the slope is inversely dependent on pressure. Least squares regression analyses of the dependence of $V_{\text {opt }}$ on molecular mass at pressures of 2.7, 1.5, and 0.88 mtorr yielded correlation coefficients of $0.87,0.89$, and 0.83 , respec-

* The strongest peak of the charge-state series in the electrospray spectra of tryptic peptides is most frequently the doubly protonated ion. In this study we have addressed only doubly charged tryptic peptide ions.

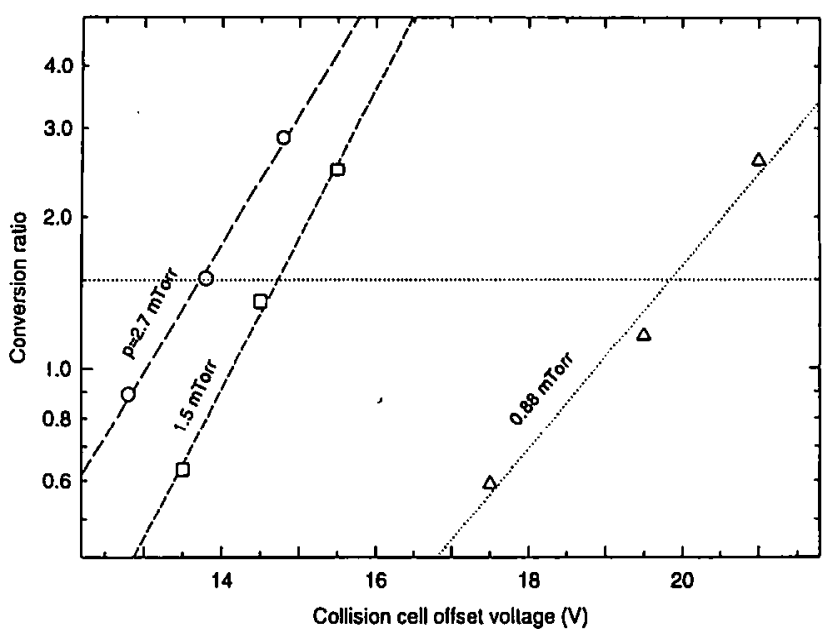

Figure 4. Conversion ratio versus offset voltage for the hexapeptide HLGLAR at three different pressures.

tively. No statistically significant difference was observed when the regression analysis was carried out separately for sets of peptides that end in lysine and in arginine. It is noteworthy that the points for any given peptide deviate from the best fit in the same direction

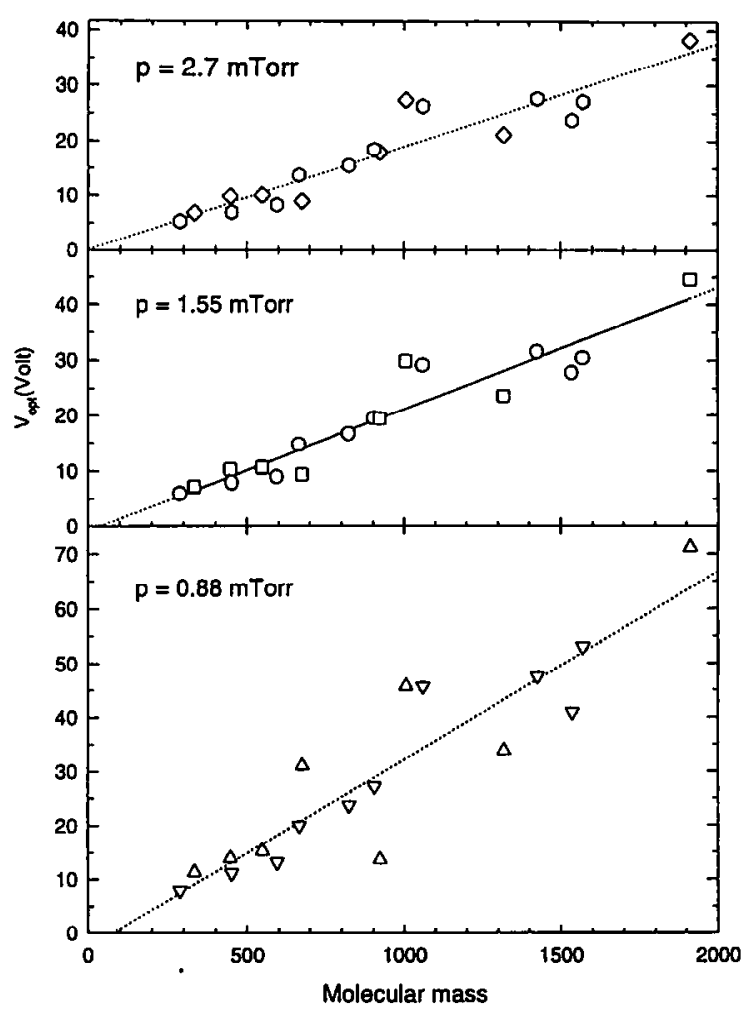

Figure 5. The dependence of the optimal acceleration voltage in CID of doubly charged ions of tryptic peptides on the molecular weight of the peptide. Hexagons, circles, and downward triangles denote arginine carboxyl termini, other symbols denote lysine carboxyl termini. The lines represent a linear regression that used all 18 data points (slopes found were 0.019, 0.022, and $0.035 \mathrm{~V} / \mathrm{u}$ and the correlation coefficients $r^{2}$ found were 0.87 , 0.89 , and 0.83 at Ar collision gas pressures of 2.7, 1.5, and 0.88 mtorr, respectively). 
for all three pressures measured. This finding indicates that the deviations result from particular molecular properties of the peptides and not from random errors. In particular, we observe a tendency for peptides that contain more than one arginine and/or lysine residue to require more energy for fragmentation than those with excess acidic residues. This observation conforms to trends that have been observed previously $[9,13]$.

Factors that effect the fragmentation of peptide ions include the center-of-mass energy available for excitation, the excitation cross section, and the number of degrees of freedom in the excited peptide. Each of these factors depends on the mass of the peptide projectile. Given these complicating factors, the observation of a simple linear dependence of $V_{\text {opt }}$ on molecular mass (Figure 5) was not predicted. It will have to be determined whether similar correlations are observed when using triple quadrupole mass spectrometers of different design.

\section{Implications for LC-MS/MS Protein Sequencing}

The observed linear dependence of the optimum collision-cell offset voltage on molecular size has significant implications for strategies used in LC-MS/MS sequencing of proteins. The simplest strategy involves tandem mass spectrometric analysis of each eluting peptide at a common, pre-selected collision energy and pressure $[1,2]$. This technique is predicted to work well if all the peptides in the mixture have approximately the same molecular mass, as occurs, for example, in sets of peptides involved in MHC antigen recognition [17]. The use of a pre-selected collision energy and pressure is sub-optimal for the collection of high quality sequence information from peptides that have a wide range of masses, for example, peptide mixtures obtained from tryptic digestion of proteins. One remedy for this problem involves the use of three collision energies for each peptide ion $[18,19]$. This remedy is accomplished by stepping the collision-cell offset voltage through three voltages for each eluting species. This strategy has the advantage of simplicity but the disadvantage that optimal information gathering only occurs during one third of the time during which a peak elutes.

The linear correlation between $V_{\text {opt }}$ and molecular mass observed in the present work suggests a better strategy for LC-MS/MS than the approaches described in the previous paragraph. Our proposed strategy involves three steps: (1) Use one to two scans in the single mass spectrometry mode (with argon in the collision cell and the collision-cell voltage set to zero) to determine the mass-to-charge ratio of the major component at the onset of elution of a highperformance liquid chromatography (HPLC) peak. (2) Use real-time computer control to set the collision offset voltage to $V_{\text {opt }}$ interpolated for the mass of interest from our simple linear relationship. (3) Average the CID spectra scanned under optimal conditions for the rest of the eluting peak. This three-step process is repeated for each eluting peak. It is imperative that the first two steps be accomplished in a few seconds. Our observation that the parent ion signal is not attenuated by the presence of the collision gas provided that the collision-cell offset voltage is kept small (data not shown) makes the first step fast by avoiding a time-consuming evacuation of the collision cell. The fitting constants for the straight line relating $V_{\text {opt }}$ and peptide mass must be predetermined with two or three standard peptides for any given mass spectrometer at the collision pressure to be used. It is envisaged that the collision-cell offset voltage will be adjusted rapidly to the appropriate values of $V_{\text {opt }}$ as the various peaks elute from the HPLC so that the bulk of the eluting peptides can be utilized for tandem mass spectrometry analysis. Within the validity of the predetermined linear correlation, our approach should yield optimal sequence information.

\section{Acknowledgment}

This work was supported by NIH Grant RR00862.

\section{References}

1. Covey, T. R.; Huang, E. C.; Henion, J. D. Anal. Chem. 1991, 63, 1193.

2. Kassel, D. B.; Shushan, B., Sakuma, T.; Salzmann, J.-P. Anal. Chem. 1994, 66, 236.

3. Hunt, D. F.; Yates, J. R., Shabanowitz, J.; Winston, S.; Hauer, C. R. Proc. Natl. Acad. U.S. A. 1986, 83, 6233.

4. Barinaga, C. J.; Edmonds, C. G.; Udseth, H. R.; Smith, R. D. Rapid Commun. Mass Spectrom. 1989, 3, 160.

5. Hunt, D. F.; Zhu, N.-Z.; Shabanowitz, J. Rapid Commun. Mass Spectrom. 1989, 3, 122.

6. Roepstorff, P.; Fohlman, J. Biomed. Mass Spectrom. 1984, 11, 601.

7. Biemann, K., Annual Rev. Biochem. 1992, 61, 977.

8. Tang, X.J.; Thibault, P.; Boyd, R. K. Anal. Chem. 1993, 65, 2824.

9. Bean, M. F.; Carr, S. A.; Thorne, G. C.; Reilly, M. S.; Gaskell, S. J. Anal. Chem. 1991, 63, 1473.

10. McCormack, A. L.; Eng, J. K.; Yates, J. R. Methods (San Diego), 1994, 6, 274.

11. Bordas-Nagy, J.; Despeyroux, D.; Jennings, K. R. J. Am. Soc. Mass Spectrom. 1992, 3, 502.

12. Curcuruto, O.; Hamdan, H. Rapid Comm. Mass Spectrom. 1993, 7, 989.

13. Jones, J. L.; Dongre, A. R.; Somogyi, A., Wysocki, V. H. J. Am. Chem. Soc. 1994, 116, 8368.

14. Bordas-Nagy, J.; Jennings, K. R. Int. J. Mass Spectrom. Ion Processes, 1990, 100, 105.

15. Morris, M.; Thibault, P.; Boyd, R. K. I. Am. Soc. Mass Spectrom. 1994, 5, 1042.

16. Thomson, B. A.; Douglas, D. J.; Corr, J. J., Hager, J. W.; Joliffe, C. L. Anal. Chem. 1995, 67, 1696.

17. Hunt D. F.; Henderson, R. A.; Shabanowitz, J.; Sakaguchi, K.; Michel, H.; Sevilir, N.; Cox, A. L.; Appella, E., Engelhard, V. H. Science 1992, 255, 1261.

18. Carr, S. A.; Huddleston, M. J.; Bean, M. F. Protein Science $1993,2,183$.

19. Mylchreest, I.; Wheeler, K.; Campbell, C. Finnigan Corporation, Application Report 240, 1995. 